\title{
Monitoring Fetal Heart Rate during Labor: A Comparison of Three Methods
}

\author{
Tammy Y. Euliano, ${ }^{1,2}$ Shalom Darmanjian, ${ }^{3}$ Minh Tam Nguyen, ${ }^{3}$ \\ John D. Busowski, ${ }^{4}$ Neil Euliano, ${ }^{3}$ and Anthony R. Gregg ${ }^{2}$ \\ ${ }^{1}$ Department of Anesthesiology, University of Florida College of Medicine, Gainesville, FL, USA \\ ${ }^{2}$ Department of Obstetrics and Gynecology, University of Florida College of Medicine, Gainesville, FL, USA \\ ${ }^{3}$ OBMedical, Jonesville, FL, USA \\ ${ }^{4}$ Winnie Palmer Hospital for Women \& Babies, Orlando, FL, USA
}

Correspondence should be addressed to Tammy Y. Euliano; teuliano@ufl.edu

Received 27 July 2016; Revised 8 February 2017; Accepted 12 February 2017; Published 14 March 2017

Academic Editor: Fabio Facchinetti

Copyright (C) 2017 Tammy Y. Euliano et al. This is an open access article distributed under the Creative Commons Attribution License, which permits unrestricted use, distribution, and reproduction in any medium, provided the original work is properly cited.

\begin{abstract}
The purpose of the study was to compare the accuracy of a noninvasive fetal heart rate monitor with that of ultrasound, using a fetal scalp electrode as the gold standard, in laboring women of varying body habitus, throughout labor and delivery. Laboring women requiring fetal scalp electrode were monitored simultaneously with the investigational device (noninvasive fetal ECG), ultrasound, and fetal scalp electrode. An algorithm extracted the fetal heart rate from the noninvasive fetal ECG signal. Each noninvasive device recording was compared with fetal scalp electrode with regard to reliability by positive percent agreement and accuracy by root mean squared error. Seventy-one women were included in this analysis. Positive percent agreement was $83.4 \pm 15.4 \%$ for noninvasive fetal ECG and $62.4 \pm 26.7 \%$ for ultrasound. The root mean squared error compared with fetal scalp electrode-derived fetal heart rate was $4.8 \pm 2.0 \mathrm{bpm}$ for noninvasive fetal ECG and $14.3 \pm 8.2 \mathrm{bpm}$ for ultrasound. The superiority of noninvasive fetal ECG was maintained for stages 1 and 2 of labor and increases in body mass index. Compared with fetal scalp electrode-derived fetal heart rate, noninvasive fetal ECG is more accurate and reliable than ultrasound for intrapartum monitoring for stages 1 and 2 of labor and is less affected by increasing maternal body mass index. This confirms the results of other workers in this field.
\end{abstract}

\section{Introduction}

Fetal well-being is assessed through electronic monitoring of uterine activity and fetal heart rate (FHR) patterns. These signals can be obtained noninvasively, traditionally through tocodynamometry and Doppler ultrasound (US), or invasively with an intrauterine pressure catheter (IUPC) and fetal scalp electrode (FSE). The latter methods require ruptured membranes and entail some small risk of infection $[1,2]$ and bleeding [3] but generally suffer less signal loss and provide additional information: the IUPC provides quantitative intrauterine pressure, and the fetal ECG can be obtained from the FSE.

International Federation of Gynecology and Obstetrics recommendations for FHR monitoring [4] include "that the baseline and the variability may be clearly read off at least $80 \%$ of the time." That target is often not reached with US $[5,6]$, particularly in the obese parturient [7].

An alternative noninvasive method entails detecting both uterine activity [electrohysterogram (EHG)] and FHR [noninvasive fetal ECG] via electrodes located on the maternal abdomen. This technology is less dependent on proximity of the sensor to the target and therefore functions regardless of the patient's body habitus.

We previously demonstrated the superiority of EHG over tocodynamometry for uterine activity monitoring, using IUPC as the gold standard [8]. For the current study, we used a similar methodology to compare the abdominal fetal ECG (afECG) with US, using FSE as the gold standard. 


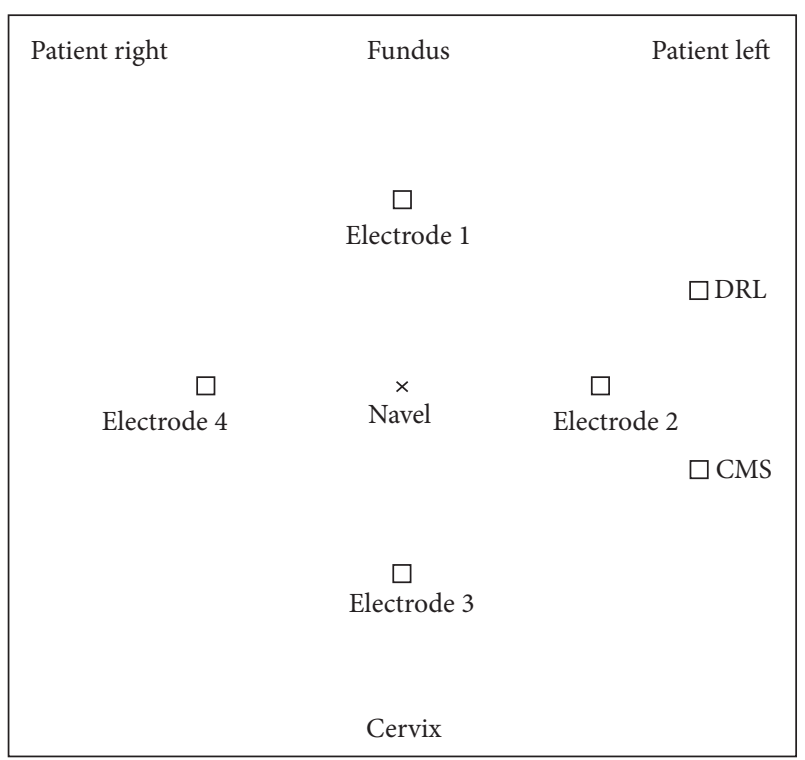

Figure 1: Location of electrodes.

\section{Materials and Methods}

This study is an analysis of the FHR data from an unpublished larger data collection, using only those patients monitored simultaneously with all three FHR devices. It was conducted at two Florida hospitals: UF Health, the University of Florida's teaching hospital (Gainesville, FL), and Winnie Palmer Hospital for Women \& Babies (Orlando, FL). The protocol was approved by the Institutional Review Boards at both institutions (UF\# 346-2010, WP\# 13.153.09) and each subject provided written, informed consent. Adult women admitted to the labor and delivery suites at term ( $\geq 37$ weeks' gestation), in active labor with a singleton fetus in cephalic presentation, without bleeding, uterine scar, or evidence of chorioamnionitis, and with FSE in place for obstetric indication (as determined by the attending obstetrician) were eligible for inclusion.

Following skin preparation by gentle rubbing with abrasive gel (OneStep AbrasivPlus, Liquimedics Pty Ltd., Germany), six 3-cm ${ }^{2} \mathrm{Ag} / \mathrm{AgCl}_{2}$ electrodes (T-00-S; Ambu; Glen Burnie, MD) were attached to the maternal abdomen (Figure 1). The electrodes were connected to the amplifier in a monopolar fashion with common reference and common mode rejection leads on the left side of the patient's abdomen to reduce $60 \mathrm{~Hz}$ environmental noise. Electrode positions were modified slightly for each patient, as required by the location of the tocodynamometer and US FHR monitor. Impedance of each electrode was measured (as compared with the reference) (General Devices $10 \mathrm{~Hz}$ EIM-105 PrepCheck; Ridgefield, NJ). Skin preparation was repeated as needed at each site until the measured impedance was below $10 \mathrm{k} \Omega$ where possible.

In addition to the experimental system, data from each patient included FHR from the maternal-fetal monitor: US
(Corometrics 250 series, GE Medical Systems, Waukesha, WI) and FSE (Corometrics at UF Health, and Avalon FM50, Philips Healthcare; Andover, MA at Winnie Palmer) sampled at $4 \mathrm{~Hz}$ with 8 -bit resolution, upsampled to $8 \mathrm{~Hz}$ to match other signals from the monitors. These cardiotocographs reported the US- and FSE-derived FHR.

The signals recorded from the electrodes were fed to the custom built, four-channel high-resolution, low-noise unipolar amplifier based on the TI ADS family of ECG/EEG amplifiers. All four signals were measured with respect to the reference electrode. The amplifier design employed driven right-leg circuitry (derived from a combination of the four channels) to reduce common mode noise. The amplifier $3 \mathrm{~dB}$ bandwidth was 0.05 to $250 \mathrm{~Hz}$. Data from four abdominal channels were sampled at $500 \mathrm{~Hz}$ with 24-bit resolution.

Because the muscle activity of the uterus (EHG) differs in frequency from the maternal and fetal heart rates, a simple frequency-selective filtering technique can separate the signals, allowing for the contraction-monitoring algorithm and the FHR algorithm to be largely independent. The maternal and fetal heart rates, however, overlap in frequency. The Mermaid algorithm is in a class called blind source separation or independent component analysis. These algorithms allow for the separation of overlapping signals as long as they are created by independent sources. The algorithm employed requires at least one electrode for each independent source and uses small differences in each electrode and correlation between the channels and sources, to separate the mixed signals. This occurs in real time [9].

Four abdominal electrode signals are input to the system from the hardware described above. These signals are first preprocessed and filtered with a bandpass filter between 1 and $30 \mathrm{~Hz}$ to remove noise and the EHG signal. Next, the Mermaid algorithm finds the four largest independent signal sources in the mixed signals (e.g., maternal ECG, fetal ECG, breathing, muscle noise, and other noises). A second algorithm then selects the channel with properties expected in the fetal ECG. A trust factor reports how well the system was able to extract the desired signals.

Data from the FSE, US (using a second electronic fetal monitoring unit), and afECG were collected simultaneously via a laptop computer. The data collector was instructed to attempt to reposition the US to obtain a reliable signal in all subjects. Clinicians were blinded to all but the FSE output for FHR monitoring and intervention.

Reliability was assessed by the positive percent agreement (PPA), the percentage of time the noninvasive device (US or afECG) generated FHR within $10 \%$ of the FHR from the FSE. FHR estimation of afECG utilized a 10-beat average to approximate the averaging from the other devices. This was calculated for each subject and averaged. Accuracy of each FHR output was estimated using the root mean squared error (RMSE), the instantaneous FHR differences between comparator and FSE. Subject populations at each study site were compared using a paired $t$-test with a significance level of 0.025 . 
TABLE 1: Demographics comparison between sites.

\begin{tabular}{lccc}
\hline Demographic variables & Total & UF Health & Winnie Palmer \\
$(n=71)$ & $(n=10)$ & $281)$ & $28.3 \pm 6.2$ \\
\hline Age (years) & $27.8 \pm 6.2$ & $25.2 \pm 5.6$ & $39.0 \pm 1.1$ \\
Gestational age (weeks) & $39.1 \pm 1.3$ & $39.5 \pm 2.0$ & $34.15 \pm 8.2$ \\
Body mass index & $35.1 \pm 8.3$ & $38.2 \pm 8.7$ & 0.23 \\
\hline
\end{tabular}

Monitoring uterine activity during labor.

TABLE 2: Performance of abdominal fetal ECG and ultrasound compared to fetal scalp electrode.

\begin{tabular}{|c|c|c|c|c|c|c|}
\hline \multirow{2}{*}{ All stages } & \multicolumn{3}{|c|}{ All subjects } & \multicolumn{3}{|c|}{ Obese subjects: $\mathrm{BMI} \geq 30$} \\
\hline & Ultrasound & afECG & $p$ & Ultrasound & afECG & $p$ \\
\hline & \multicolumn{3}{|c|}{$(n=71)$} & \multicolumn{3}{|c|}{$(n=51)$} \\
\hline PPA (\%) & $62.4 \pm 26.5$ & $83.4 \pm 15.4$ & $<0.0001$ & $58.1 \pm 25.8$ & $84.4 \pm 14.6$ & $<0.0001$ \\
\hline RMSE (bpm) & $14.3 \pm 8.2$ & $4.8 \pm 2.0$ & $<0.0001$ & $15.6 \pm 8.1$ & $4.8 \pm 2.1$ & $<0.0001$ \\
\hline Stage 1 & \multicolumn{3}{|c|}{$(n=48)$} & \multicolumn{3}{|c|}{$(n=36)$} \\
\hline PPA (\%) & $61.3 \pm 29.6$ & $86.3 \pm 14.7$ & $<0.0001$ & $55.6 \pm 28.8$ & $79.1 \pm 13.3$ & $<0.0001$ \\
\hline RMSE (bpm) & $13.6 \pm 7.9$ & $5.0 \pm 2.0$ & $<0.0001$ & $15.5 \pm 8.1$ & $4.9 \pm 2.0$ & $<0.0001$ \\
\hline Stage 2 & \multicolumn{3}{|c|}{$(n=23)$} & \multicolumn{3}{|c|}{$(n=15)$} \\
\hline PPA (\%) & $64.5 \pm 18.5$ & $77.5 \pm 15.1$ & $<0.003$ & $64.0 \pm 15.3$ & $79.1 \pm 13.3$ & $<0.007$ \\
\hline RMSE(bpm) & $15.8 \pm 8.4$ & $5.0 \pm 2.0$ & $<0.0001$ & $15.7 \pm 7.9$ & $4.9 \pm 2.0$ & $<0.0002$ \\
\hline
\end{tabular}

Monitoring uterine activity during labor.

\section{Results}

Patient characteristics did not differ between sites (Table 1).

The average PPA for afECG, $83.4 \%$, exceeded that for US (62.4\%, $p<0.0001$, Table 2$)$. That superiority persisted in both first $(p<0.0001)$ and second $(p<0.003)$ stages of labor for all subjects. Furthermore, afECG was more accurate, with a mean RMSE of $4.8 \mathrm{bpm}$ compared to 14.3 for US ( $p<$ $0.0001)$. In obese parturients $\left(\mathrm{BMI}>30 \mathrm{~kg} / \mathrm{m}^{2}\right)$, the afECG again outperformed US in both PPA (84.4\% versus $58.1 \%$, $p<0.0001)$ and accuracy (4.8 versus $15.6 \mathrm{bpm}, p<0.0001)$. Furthermore, afECG showed no drop-off in performance between normal weight (PPA 81.0\% \pm 17.2\%; RMSE $4.9 \pm$ $1.9 \mathrm{bpm}$ ) and obese subjects (PPA $84.4 \pm 14.6 \%$; RMSE 4.8 $\pm 2.1 \mathrm{bpm}$ ), while US performance was adversely affected by obesity: normal weight PPA 73.2\% \pm 25.2\%; RMSE $11.0 \pm$ 7.2 bpm; obese PPA 58.1\% \pm 25.8\%; RMSE $15.6 \pm 8.1 \mathrm{bpm}$.

\section{Discussion}

Doppler US is the most common method for continuous FHR monitoring and functions adequately in most situations. Its frequent regions of dropout and occasional confusion with maternal heart rate [10] complicate interpretation in the setting of a nonreassuring tracing. When external monitoring is unreliable, clinicians may artificially rupture membranes to place more dependable internal monitors. This increases the duration of ruptured membranes and risk of infection.

Failure of external US monitoring is more common in the obese population [7], comprising nearly one-third of women of child-bearing age [11]. These patients are more likely to experience complications [12-14], require internal monitoring [7], have prolonged first-stage labor [15], and undergo cesarean delivery $[14,16,17]$. The reason for the increased cesarean rate is likely multifactorial, including the slow pace of cervical dilation and the concomitant increased number of cervical examinations and need for internal monitoring, resulting in a higher rate of chorioamnionitis, which itself increases the cesarean delivery rate [18].

An alternative external monitoring system that provides reliable FHR and uterine activity regardless of maternal size may improve outcomes. In addition, the continuous display of maternal heart rate will reduce maternal-fetal heart rate confusion incidents.

Cohen et al. [19] compared US, FSE, and fECG from the maternal abdomen (afECG) via the AN24 system (Monica Healthcare Ltd., Nottingham, UK) in 75 laboring women with a protocol similar to that reported here. PPA was reported as the percentage of time the external monitor reported FHR within $10 \%$ of that derived from the FSE and was superior for the afECG device ( $81.7 \%$ versus $73 \%$ for US). This superiority persisted in analysis of both first- and second-stage labor (PPA afECG $84.9 \%$ and $71.9 \%$ versus US $74.7 \%$ and $61.7 \%$, for first and second stage, resp.). The afECG also demonstrated improved accuracy with RMSE of $5.3 \pm 2.4 \mathrm{bpm}$ versus $10.9 \pm$ $5.8 \mathrm{bpm}$ for US.

The success of their afECG compares favorably with our results, though US underperformed in our study. In both studies, FSE was placed for obstetric indication. Cohen states, "in all cases [FSE was placed] because the external tracing was abnormal." Whether abnormal in this case includes high dropout is unclear. Although we did not record the indication in our study, subjects were more likely to have an unacceptable rate of dropout with US, thus biasing our results 
against that device. Furthermore, in both studies, subjects were monitored clinically by FSE. Although both protocols specified adjustments to US when it failed, it is possible that US was not optimally used during the study. Regardless, the PPA comparison of afECG with FSE is valid and proves the utility of afECG in the population where US fails.

Graatsma et al. [20] found no impact of increasing body mass index (BMI) on afECG signals with the AN24 system. Their cohort was 20- to 42-week, nonlaboring pregnant women monitored during sleep and therefore differs substantially from the active labor environment of this report.

Cohen and Hayes-Gill [21] looked specifically at the impact of BMI on the accuracy and reliability of external monitoring. In a secondary analysis of 74 parturients monitored simultaneously by all three methods (US, FSE, and AN24), they found no effect of maternal obesity on the performance of their system, while US performance "degraded directly with maternal size." In their study, nine subjects with BMI $>40$ had PPA of $81.4 \pm 23.8 \%$ for afECG. This compares to our results of $86.1 \pm 15 \%$ on 19 such patients.

In summary, we found that afECG is superior to US in both reliability and accuracy when compared to FSE in all subjects and in the obese subset. This may have clinical implications for the assessment of fetal well-being, particularly in the obese subjects, where US frequently fails to provide an adequate FHR trace.

\section{Disclosure}

These results have been presented, in part, at the University of Florida College of Medicine Celebration of Research, February 2015.

\section{Conflicts of Interest}

The husband of Tammy Y. Euliano is Chief Technical Officer and Minh Tam Nguyen and Shalom Darmanjian are employees of OBMedical. Tammy Y. Euliano owns no stock nor holds any position in the company but is listed on patents filed for some of the technology described in this paper. The authors declare that there are conflicts of interest regarding the publication of this article.

\section{Authors' Contributions}

Tammy Y. Euliano, Neil Euliano, and Shalom Darmanjian conceived and designed the experiments. John D. Busowski and Anthony R. Gregg performed the experiments. Shalom Darmanjian and Minh Tam Nguyen extracted and analyzed the data. Neil Euliano contributed materials. Tammy Y. Euliano, Shalom Darmanjian, Minh Tam Nguyen, and Anthony R. Gregg wrote the paper.

\section{Acknowledgments}

The authors would like to thank the data collector at UF Health, Teresa Lyles, Ph.D., and, at Winnie Palmer, Michele L. Real, as well as their statistician, Gary Stevens, and their editor, Corey Astrom. Finally, they would like to thank the nursing and physician staff at both hospitals. The study was supported by OBMedical and the University of Florida. The former supplied the monitoring device and funded the research coordinator and subject payments. Tammy Y. Euliano was funded at $\sim 10 \%$ salary. Tammy Y. Euliano will not benefit from the patents as they belong to her employer, the University of Florida.

\section{References}

[1] L. M. Harper, A. L. Shanks, M. G. Tuuli, K. A. Roehl, and A. G. Cahill, "The risks and benefits of internal monitors in laboring patients," American Journal of Obstetrics and Gynecology, vol. 209, no. 1, pp. 38.el-38.e6, 2013.

[2] S. F. Siddiqi and P. M. Taylor, "Necrotizing fasciitis of the scalp: a complication of fetal monitoring," American Journal of Diseases of Children, vol. 136, no. 3, pp. 226-228, 1982.

[3] R. Spernol, "Severe infantile bleeding from a vasa praevia during application of a fetal electrode following spontaneous rupture of the fetal membranes," Zeitschrift Fur Geburtshilfe und Perinatologie, vol. 185, no. 6, p. 364, 1981.

[4] The International Federation of Gynecology and Obstetrics, "Guidelines for the use of fetal monitoring," International Journal of Gynecology \& Obstetrics, vol. 25, pp. 159-167, 1987.

[5] P. C. A. M. Bakker, G. J. Colenbrander, A. A. Verstraeten, and H. P. Van Geijn, "The quality of intrapartum fetal heart rate monitoring," European Journal of Obstetrics Gynecology and Reproductive Biology, vol. 116, no. 1, pp. 22-27, 2004.

[6] J. Reinhard, B. R. Hayes-Gill, S. Schiermeier et al., "Intrapartum signal quality with external fetal heart rate monitoring: a two way trial of external Doppler CTG ultrasound and the abdominal fetal electrocardiogram," Archives of Gynecology and Obstetrics, vol. 286, no. 5, pp. 1103-1107, 2012.

[7] A. Ray, A. Hildreth, and U. I. Esen, "Morbid obesity and intrapartum care," Journal of Obstetrics and Gynaecology, vol. 28, no. 3, pp. 301-304, 2008.

[8] T. Y. Euliano, M. T. Nguyen, S. Darmanjian et al., "Monitoring uterine activity during labor: a comparison of 3 methods," American Journal of Obstetrics and Gynecology, vol. 208, no. 1, pp. 66.e1-66.e6, 2013.

[9] D. Erdogmus, K. E. Hild II, Y. N. Rao, and J. C. Príncipe, "Minimax mutual information approach for independent component analysis," Neural Computation, vol. 16, no. 6, pp. 1235-1252, 2004.

[10] T. Stampalija, M. Signaroldi, C. Mastroianni et al., "Fetal and maternal heart rate confusion during intra-partum monitoring: comparison of trans-abdominal fetal electrocardiogram and doppler telemetry," Journal of Maternal-Fetal and Neonatal Medicine, vol. 25, no. 8, pp. 1517-1520, 2012.

[11] Centers for Disease Control, NCHS Obesity Data, National Center for Health Statistics, 2014, http://obesity.procon.org/sourcefiles/NCHSDataOnObesity.pdf.

[12] R. Scott-Pillai, D. Spence, C. R. Cardwell, A. Hunter, and V. A. Holmes, "The impact of body mass index on maternal and neonatal outcomes: A Retrospective Study in a UK Obstetric Population, 2004-2011," BJOG: An International Journal of Obstetrics and Gynaecology, vol. 120, no. 8, pp. 932-939, 2013.

[13] E. F. Magann, D. A. Doherty, S. P. Chauhan, J. M. Klimpel, S. D. Huff, and J. C. Morrison, "Pregnancy, obesity, gestational weight gain, and parity as predictors of peripartum complications," Archives of Gynecology and Obstetrics, vol. 284, no. 4, pp. 827836, 2011. 
[14] W. Kabiru and B. Denise Raynor, "Obstetric outcomes associated with increase in BMI category during pregnancy," American Journal of Obstetrics and Gynecology, vol. 191, no. 3, pp. 928932, 2004.

[15] S. Carlhäll, K. Källén, and M. Blomberg, "Maternal body mass index and duration of labor," European Journal of Obstetrics \& Gynecology and Reproductive Biology, vol. 171, no. 1, pp. 49-53, 2013.

[16] M. I. Cedergren, "Non-elective caesarean delivery due to ineffective uterine contractility or due to obstructed labour in relation to maternal body mass index," European Journal of Obstetrics Gynecology and Reproductive Biology, vol. 145, no. 2, pp. 163-166, 2009.

[17] B. P. Wispelwey and E. Sheiner, "Cesarean delivery in obese women: a comprehensive review," Journal of Maternal-Fetal and Neonatal Medicine, vol. 26, no. 6, pp. 547-551, 2013.

[18] R. K. Edwards, "Chorioamnionitis and labor," Obstetrics and Gynecology Clinics of North America, vol. 32, no. 2, pp. 287-296, 2005.

[19] W. R. Cohen, S. Ommani, S. Hassan et al., "Accuracy and reliability of fetal heart rate monitoring using maternal abdominal surface electrodes," Acta Obstetricia et Gynecologica Scandinavica, vol. 91, no. 11, pp. 1306-1313, 2012.

[20] E. M. Graatsma, J. Miller, E. J. H. Mulder, C. Harman, A. A. Baschat, and G. H. A. Visser, "Maternal body mass index does not affect performance of fetal electrocardiography," American Journal of Perinatology, vol. 27, no. 7, pp. 573-577, 2010.

[21] W. R. Cohen and B. Hayes-Gill, "Influence of maternal body mass index on accuracy and reliability of external fetal monitoring techniques," Acta Obstetricia et Gynecologica Scandinavica, vol. 93, no. 6, pp. 590-595, 2014. 


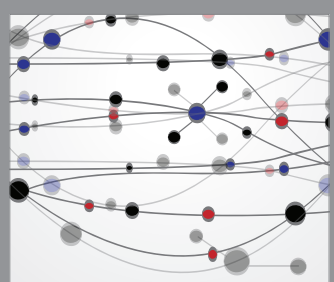

The Scientific World Journal
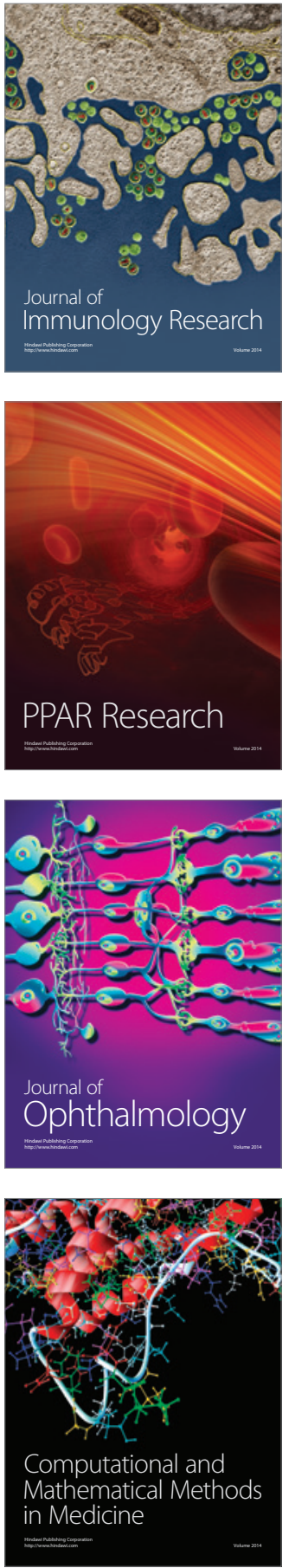

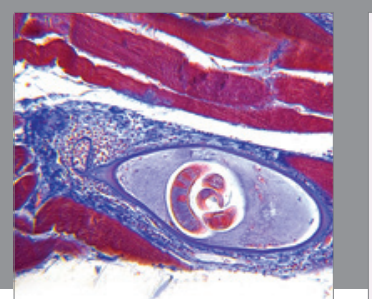

Gastroenterology Research and Practice
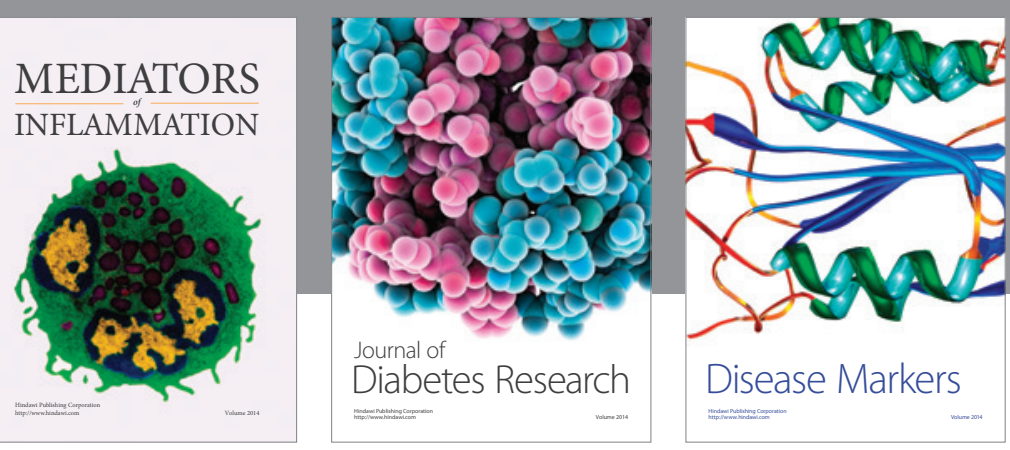

Disease Markers

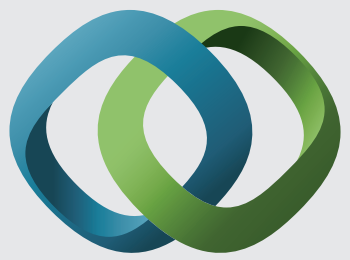

\section{Hindawi}

Submit your manuscripts at

https://www.hindawi.com
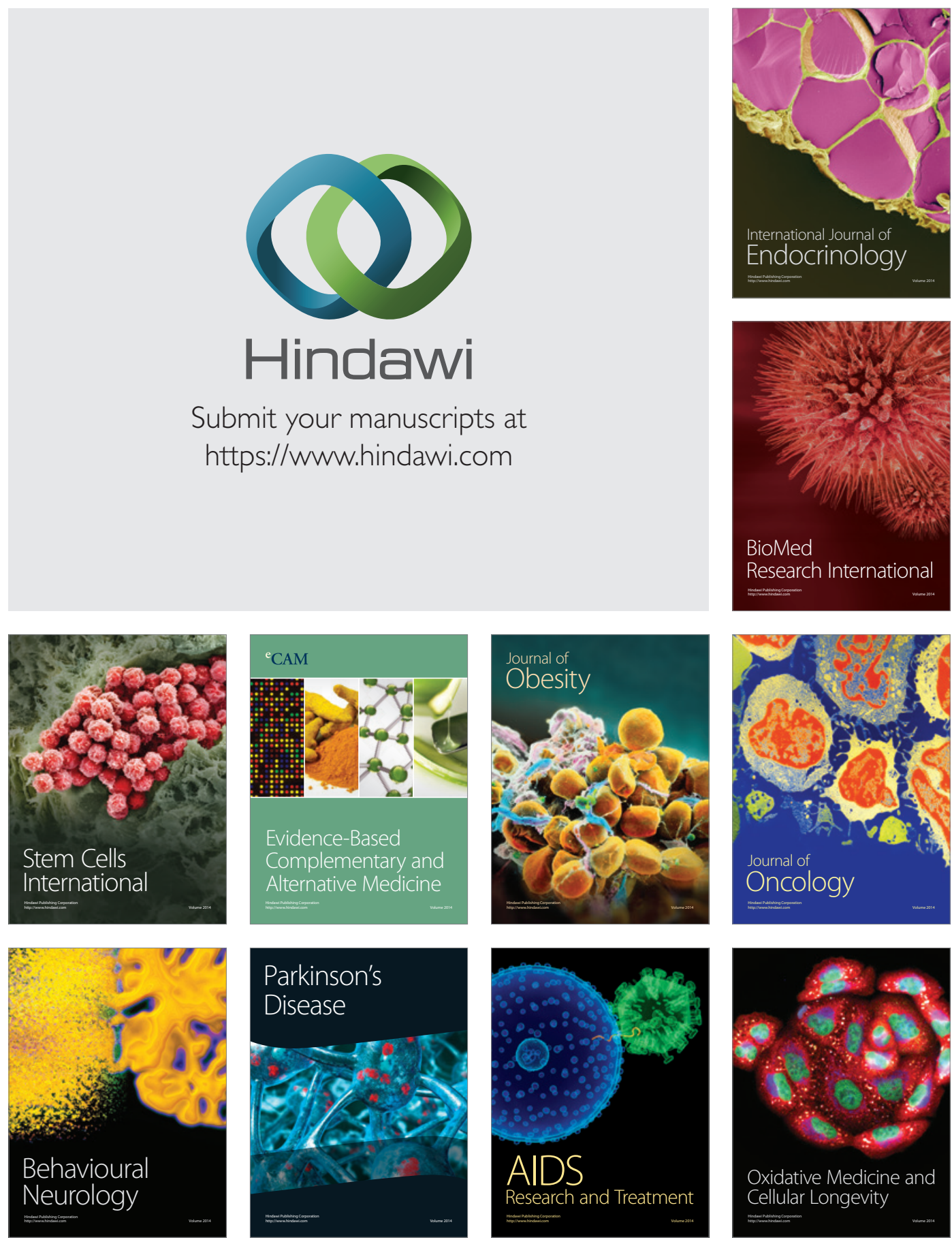